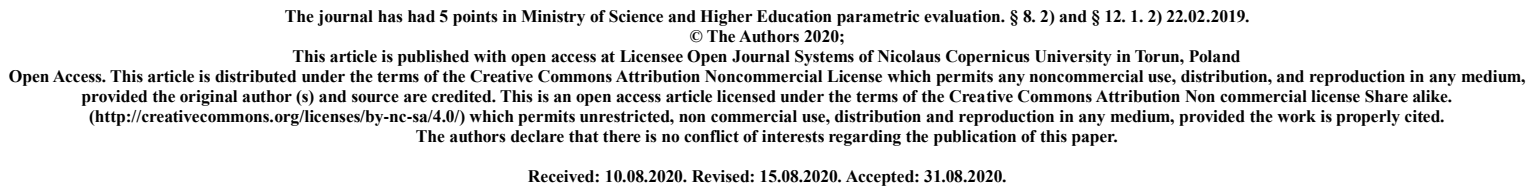

Role of the gut microbiota in human health

\author{
Aleksandra Chałupnik ${ }^{1}$, Zuzanna Chilimoniuk ${ }^{1}$, Anna Sobstyl ${ }^{1}$, \\ Maciej Dobosz ${ }^{1}$, Aleksandra Borkowska ${ }^{1}$, Malgorzata Wieteska ${ }^{2}$
}

1. Student Research Circle at the Department of Family Medicine, Medical University of Lublin Supervisor: PhD Grzegorz Mizerski

2. Student Research Circle at the Department of Epidemiology and Clinical Research Methodology, Medical University of Lublin

Corresponding author: Aleksandra Chałupnik, olachalupnik@op.pl

\title{
ORICID ID:
}

Aleksandra Chałupnik: https://orcid.org/0000-0003-4249-470X

Zuzanna Chilimoniuk: https://orcid.org/0000-0001-8261-0192

Anna Sobstyl: https://orcid.org/0000-0003-0330-5742

Maciej Dobosz: https://orcid.org/0000-0002-4413-3310

Aleksandra Borkowska: https://orcid.org/0000-0002-0950-2176

Małgorzata Wieteska: https://orcid.org/0000-0002-1566-7559

\begin{abstract}
Introduction: The human gut microbiota has become the subject of extensive research in recent years and the knowledge of the species inhabiting the gut is growing rapidly. Microbiota plays an important role in human health and diseases. It takes part in obtaining and storing energy, as well as in metabolic functions.
\end{abstract}

The aim of the study: The purpose of the study is to collect and review scientific publications about the impact of gut microbiota on human health. 
Material and method: The paper uses standard criteria as the research method. The articles used to create the reviews were published on the PubMed and Google Scholar platforms.

Description of the state of knowledge: Numerous studies suggest that composition of intestinal microbiota has impact on the long-term functioning of the human organism. Depressive disorders appear to be associated with changes in the microbiota. In depressed patients, fewer bacteria of the type Bacteroidetes and higher level of Alistipes are found. A few studies have demonstrated a link between gut microbial signatures and hypertension. Studies have shown that T2DM patients may have an altered composition of gut microbiota, mainly a decreased Bacteroidetes/ Firmicutes ratio and decrease in some functional bacteria when compared to healthy people. Intestinal microorganisms are also a key element in the proper functioning of the immune system by influencing the maturation process of the immune system's cells.

Summary: To sum up, numerous studies indicate that abnormal composition of the intestinal microflora is the cause of not only faster development of metabolic syndrome, but also other diseases. It influences the development of circulatory, nervous or immune system diseases. Its abnormal composition is influenced by many factors such as diet, lifestyle or antibiotic therapy.

Keywords: gut microbiota, health, probiotics, microorganisms

\section{INTRODUCTION}

The human gut microbiota has become the subject of extensive research in recent years and the knowledge of the species inhabiting the gut is growing rapidly [1]. Currently, there are approximately 100 billion microorganisms in the human digestive tract [2]. Microbiota plays an important role in human health and diseases. It takes part in obtaining and storing energy, as well as in metabolic functions such as fermentation or absorption of undigested carbohydrates. Intestinal microorganisms are also a key element in the proper functioning of the immune system by influencing the maturation process of the immune system's cells [3]. Numerous studies indicate that the intestinal microflora is an environmental factor affecting predisposition to obesity [4]. By modifying the ecology of intestinal microorganisms caused by dietary factors, antibiotics, probiotics or prebiotics, the relationship between the composition of intestinal microflora and obesity has also been demonstrated. Therefore, the identification of early changes in the intestinal microflora may predict the subsequent development of obesity [5].

\section{THE IMPACT OF GUT MICROBIOTA ON HUMAN HEALTH}

\section{Cardiovascular system}

Cardiovascular disease (CVD) is a class of diseases that involve the heart or blood vessels. CVD includes atherosclerosis, hypertension, heart failure, atrial fibrillation, myocardial fibrosis, cardiomyopathy [6]. Many risk factors are widely known, such as smoking, poor dietary habits, obesity, diabetes mellitus and high cholesterol, but these cannot explain all CVD incidences [7]. CVD is the main cause of death globally. 
The recently discovered contribution of gut microbiota-derived molecules in the development of heart disease and its risk factors has significantly increased attention towards the connection between our gut and heart [8]. In the study, researchers using a targeted metabolomics approach aimed at identifying plasma metabolites, have identified a novel pathway linking dietary lipid intake, intestinal microflora and atherosclerosis [9]. The pathway identified, represents a unique nutritional contribution to the pathogenesis of CVD. Researchers performed a metabolomic analysis of the plasma of patients with known atherosclerotic disease. They identified three metabolites of dietary phosphatidylcholine - choline, betaine, and, trimethylamine N-oxide (TMAO) — and showed that these phospholipid metabolites were independent predictors for the risk of a clinical vascular event. The pro-atherogenic gut flora-generated metabolite, TMAO, is formed in a two-step process initiated by gut flora-dependent cleavage of a trimethylamine species generating the precursor TMA, and subsequent oxidation by FMO3 [9]. Results indicate that both environmental exposure (dietary lipid) and microbial flora participate in TMAO production and the atherogenic macrophage phenotype and are consistent with the view that trimethylamine, the precursor of TMAO is produced by gut microbiota. It confirms earlier observations in germ-free mice on the bacterial origin of this methylamine [10]. The incidence of cardiovascular events increases with the elevated levels of TMAO in patients with acute coronary syndrome whose troponin $\mathrm{T}$ is negative [11]. Gut microbiome, especially Synergistetes phylum, Spirochaetes phylum, Lachnospiraceae family, Syntrophomonadaceae family and Tissierella Soehngeniagenus, present a higher trend in acute MI animal models [12]. TMAO is associated with increased risk of adverse cardiovascular events including MI in stroke patients [13]. It can predict adverse outcomes of all-cause mortality or reinfarction 2 years after MI [14]. A few studies have demonstrated a link between gut microbial signatures and hypertension. It has been illustrated that intestinal flora facilitate vascular dysfunction induced by angiotensin-II (Ang-II ) by promoting systemic inflammation responses that lead to high blood pressure [15]. One study found that Klebsiella, Clostridium and Streptococcus are more abundant in hypertensive patients [16]. TMAO does not affect blood pressure directly but prolongs the haemodynamic effects of Ang-II [17]. In heart failure (HF) patients, reduced cardiac output and blood redistribution result in a decrease in intestinal perfusion and disruption of the intestinal barrier. Thus, the microbiota and endotoxins translocate into the blood circulation, aggravate systemic inflammation and exacerbate HF [18]. Elevated TMAO level is relevant to the severity of HF and indicates a worse prognosis [19]. A growing body of data reveal a strong association between TMAO and myocardial fibrosis. TMAO can aggravate myocardial interstitial fibrosis and perivascular fibrosis, damaging heart compliance and function [20]. TMAO can reverse the protective effect of voluntary exercise on myocardial fibrosis induced by high sugar and fat diet [21]. The mechanism by which TMAO aggravates myocardial fibrosis is not fully understood. Moreover all these results suggest that an appropriately designed probiotic intervention may serve as a therapeutic strategy for CVD. 


\section{The gut brain-axis}

Gut bacteria have the ability to communicate with the CNS in two ways by numerous mechanisms: changes in the composition of the microbiota, stimulation of the immune system, through the neural pathways (vagus nerve), by tryptophan metabolism, the endocrine response of the gut, and metabolites produced by bacteria [22-26]. The gut microbiota affects the central nervous system (CNS) through ability to synthesize and secrete several of neuroactive molecules such as acetylcholine, catecholamines, gamma-aminobutyric acid, histamine, melatonin and serotonin (5-hydroxytryptamine) [27]. CNS functions can also be influenced by short-chain one's fatty acids, which are fermentation products of bicarbonate and have the ability to penetrate the systemic circulation [28]. Moreover, microbiota of the intestine secretes neurotrophins and proteins, e.g. neurotrophic origin factor brain (BDNF), synaptophysin, postsynaptic protein density 95 (PSD-95). Information of hormonal and neuronal changes, changes in the composition of the intestinal tract biota passes straight to the brain through the vagus nerve that forms direct connection between the digestive system and the brain [29]. These interactions can also interact to the pathogenesis of numerous disorders, the etiopathogenesis of which has a component inflammatory - mood disorders, ADHD, autism [30]. Chrobak et al. describe that slight inflammation may play an important role in the pathophysiology of depression and disorder [31]. Stress is one of the strongest factors in everyday life life that affect the composition of the gut microbiota. Microbiota of the intestines are influenced by both emotional and physiological stress [32]. Dinan and Cryan showed that in healthy students it occurs in samples during periods of increased stress less Lactobacillus bacteria in stool compared to collected samples in a less stressful period [33].

\section{Neuropsychiatric diseases:}

While writing about neuropsychiatric diseases, it should be mentioned that the intestinal microbiota has the ability to convert undigested carbohydrates to short-chain carbohydrates fatty acids (SCFA) such as acetate, propionate and butyrate. These short-chain fatty acids can bind and activate the associated receptors with G proteins (GPR 41 and GPR 43) on intestinal epithelial cells, which leads to secretion of peptide YY (PYY) which inhibits gastrointestinal motility and delays intestinal transit [34]. SCFAs also show the ability to activate the receptors GPR 120, which improve glucose metabolism, GLP-1 release and maintenance of immune homeostasis (reduction of inflammation). Moreover, some researches show that propionate may induce autistic-like behavior in rats [35]. Autism (ASD) is one of the neuropsychological disorders in which microbiota plays a key role. Recent studies have shown that the microbiota in patients diagnosed with autism differs from the control group [36]. The number of Clostridium was significantly increased in group of autistic patients. Further evidence for the role of microbiota in autism pathogenesis was obtained by observing improvements in behavior and skills communication after antibiotics and probiotics treatment [37]. Depressive disorders, which are the most commonly diagnosed psychiatric disorders, also appear to be associated with changes in the microbiota. In depressed patients, fewer bacteria of the type Bacteroidetes and higher level of Alistipes are found [38]. Increasing numbers of Alistipes have been observed in others diseases such as, for instance, chronic fatigue syndrome [39]. The quantity and quality of the gut microbiota can also influence the presence of mood changes and anxiety disorders. 
Anxiety disorders commonly coexist with irritable bowel syndrome [40]. In a study by Rao et al. supplementation L. casei Shirota decreases the incidence of anxiety disorders in patients with chronic fatigue syndrome. The gut microbiota seems to play a significant role in the pathogenesis of many diseases, but the detailed understanding of the mechanisms composing this phenomenon requires further research which may enable future development of new treatments for these symptoms [41].

\section{Parkinson's disease}

Parkinson's disease (PD) is a progressive, neurodegenerative disorder of the CNS. This condition is characterized by the formation of Lewy bodies, made of alpha-synuclein in the substantia nigra in the midbrain. Loss of dopaminergic neurons results in motor symptoms such as akinesia, gait disorder, and resting tremor [42, 43]. Studies have shown that dysbiosis of gut microbiota plays an important role in progression of PD [44-47]. It was found that stool of PD patients contained decreased amount of Prevotellaceace species and increased amount of Enterobacteriaceae species [48]. Lower abundance of Prevotellaceace leads to excessive production of $\alpha$-synuclein in the colon and even support its abnormal folding [49]. Enterobacteriaceae overgrowth leads to an increased lipopolysaccharide (LPS) levels. LPS disrupts the intestinal epithelial barrier, penetrates the blood system, where it induces systemic inflammation and cytokine synthesis [50]. Inflammation promotes destruction of the blood-brain barrier, which determines the formation of Lewy bodies and destruction of dopaminergic neurons [51]. Additional reports also point to an increased amount of other pro-inflammatory bacteria such as Ralstonia, Proteobacteria or Enteococcaceae as well as to decreased amount of Blautia, Coprococcus, Roseburia and Faecalibacterium, that are considered as an antiinflammatory bacterium [52]. Probiotics containing bifidobacteria and lactobacilli are reported to reverse the PD-like condition and the probiotic bacterium Bacillus spp. may convert LTyrosine into L-DOPA which is converted to dopamine $[53,54]$.

\section{Alzheimer disease}

Alzheimer's disease (AD) is a neurodegenerative disorder characterized by formation of $\beta$-amyloid plaque and neurofibrillary tangles of hyperphosphorylated tau. This syndrome manifests as memory, language and behavior impairments which leads to loss of performing basic activities of daily living [55]. It has been shown that the diversity and composition of the gut microbiota of healthy people are significantly different than in patients with Alzheimer's disease [56]. A 2017 study reports that the microbiome of AD patients contains significantly less Firmicutes and Actinobacteria, and significantly more Bacteroidetes [57]. Bacterial metabolites induce inflammation that increases intestinal permeability and blood-brain barrier, which apromotes the production of beta-amyloid in the brain [58-60]. $\beta$-N-methylaminoL-alanine (BMAA) is one of the neurotoxins produced by intestinal cyanobacterias which may cause neurodegeneration and cognitive impairment [61]. LPS induces the formation of $A \beta$ fibrils which are a potent inducer of NF- $\kappa B$ known to be involved in neuroinflammation in the AD brain $[62,63]$. As in $\mathrm{PD}$, probiotic supplementation may be protective in AD. A randomized, double-blind controlled clinical trial showed a positive effect of the combination of Lactobacillus acidophilus, Lactobacillus casei, Lactobacillus bifidum, Lactobacillus fermentum on cognitive function in AD patients [64]. 
The supply of Bifidobacterium may lead to a decrease in the level of pro-inflammatory cytokines TNF- $\alpha$, IL-5, IL-6, IL-1 $\beta$ and IL-8 in the serum [65].

\section{Type 1 diabetes}

Type 1 diabetes (T1D) is one of the most common chronic diseases of childhood and the second most common autoimmune disease among children. However, it can be recognized at any age. T1D is accompanied by many complications and has life-long morbidity [66, 67]. It is thought to be precipitated by T-cell-mediated destruction of insulin-producing pancreatic $\beta$ cells. As a result, insufficient insulin production and its deficiency disrupts glucose uptake into tissues leading to the development of life-threatening health complications [68]. The development of T1D is associated with various factors, such as diet, genome, and intestinal microbiota. Gut microbiota is thought to play an important role in stimulating the development of the immune system [69]. There are different mechanisms that may explain the role of the gut microbiota in the pathogenesis of T1D [67]. T1D patients are exposed to increased mucin degradation by bacteria, which results in decreased integrity and increased permeability of the intestinal mucosa. As a result, bacteria penetrate the intestinal mucosa and the immune system is stimulated, which leads to the production of antibodies against them [70]. Additionally, certain groups of bacteria can alter the integrity of the mucosa by affecting zonulin, which is a protein responsible for modulating the intercellular junction and the passage of macromolecules through them [71]. Moreover, study conducted by Cardwell's team showed that the risk of T1D onset in childhood is higher in children delivered by Caesarean section [72]. One of the plausible explanations for this phenomenon is difference in gut microbiotic composition between vaginally born children and children born by Caesarean section. In second case, infants were first exposed to bacteria originating from the hospital environment rather than to maternal bacteria [73].

\section{Type 2 diabetes}

Type 2 diabetes (T2DM) is the most popular form of diabetes mellitus involving disorders of insulin action and insulin secretion. T2DM patients, which accounts for approximately $90-95 \%$ of cases, are at risk of developing macrovascular and microvascular complications $[74,75]$. In addition to the main risk factors such as sedentary lifestyle, poor diet and obesity, some research indicates an increasing role of the intestinal microbiota in the development of T2DM [76]. Studies have shown that T2DM patients may have an altered composition of gut microbiota, mainly a decreased Bacteroidetes/ Firmicutes ratio and decrease in some functional bacteria such as Bifidobacteria when compared to healthy people. The Bacteroidetes/ Firmicutes ratio is an environmental factor that provides genetic material for the increased ability to obtain energy from diet. Moreover, an increase in various opportunistic pathogens and also in endotoxins-producing gram-negative bacteria was discovered [74,77]. Additionally, gut-derived bacterial inflammatory molecules such as lipopolysaccharide and peptidoglycans are believed to accelerate inflammation in T2DM when they accumulate in the intestine [74]. According to Sabatino et al., the reduction in butyrate-producing bacteria such as Roseburia intestinalis and Faecalibacterium prausnitzii is characteristic for T2DM patients [78]. 
Moreover, Furet et al. found that the proportion of Faecalibacterium prausnitzii species is directly related to a reduction in low-grade inflammation following intervention in type 2 diabetes, regardless of caloric intake. It makes F. prausnitziias a potential microbial target used in glucose homeostasis control in T2DM patients [79].

\section{SUMMARY}

To sum up, numerous studies indicate that abnormal composition of the intestinal microflora is the cause of not only faster development of metabolic syndrome, but also other diseases. It influences the development of circulatory, nervous or immune system diseases. Its abnormal composition is influenced by many factors such as diet, lifestyle or antibiotic therapy. The range of inter-personal variability in the composition of microbiota in the population has also become apparent and probably affects individual reactions to the administration of drugs and diet manipulation. Probiotics and prebiotics are increasingly being used to change the composition of the microflora. However, there is still no evidence of their effectiveness when used for a long time. 


\section{References:}

1. Valdes A, Walter J, Segal E, Tim D. Role of the gut microbiota in nutrition and health. Science and Politics of Nutrition. BMJ 2018;361:k2179.

2. Clemente J, Ursell L, Wegener Parfrey L, Knight R. The Impact of the Gut Microbiota on Human Health: An Integrative View. Volume 148, Issue 6, 16 March 2012, Pages 12581270 .

3. Flint H, Scott K, Louis P, Duncan S. The role of the gut microbiota in nutrition and health. Nature Reviews Gastroenterology \& Hepatology volume 9, pages577-589(2012).

4. Boulangé C, Neves AL, Chilloux J, Nicholson JK, Dumas ME. Impact of the gut microbiota on inflammation, obesity, and metabolic disease. Genome Med. 2016; 8: 42.

5. Festi D, Schiumerini R, Eusebi LH, Marasco G, Taddia M, Colecchia A. Gut microbiota and metabolic syndrome. World J Gastroenterol. 2014 Nov 21; 20(43): 16079-16094.

6. Puska P, Norrving B (2011). Global Atlas on Cardiovascular Disease Prevention and Control World Health Organization in collaboration with the World Heart Federation and the World Stroke Organization. pp. 3-18.

7. Writing Group M, Mozaffarian D, Benjamin EJ, Go AS, Arnett DK, Blaha MJ, et al. Heart Disease and Stroke Statistics-2016 Update: A Report From the American Heart Association. Circulation. 2016.

8. Koeth RA, Wang Z, Levison BS, Buffa JA, Org E, Sheehy BT, Britt EB, Fu X, Wu Y, Li L, Smith JD, DiDonato JA, Chen J, Li H, Wu GD, Lewis JD, Warrier M, Brown JM, Krauss RM, Tang WH, Bushman FD, Lusis AJ, Hazen SL „Intestinal microbiota metabolism of L-carnitine, a nutrient in red meat, promotes atherosclerosis." Nat Med. 2013 May; 19(5):576-85.

9. Wang, Z., Klipfell, E., Bennett, B. J., Koeth, R., Levison, B. S., DuGar, B., ... Hazen, S. L. (2011). Gut flora metabolism of phosphatidylcholine promotes cardiovascular disease. Nature, 472(7341), 57-63.

10. Al-Waiz M, Mikov M, Mitchell SC, Smith RC. The exogenous origin of trimethylamine in the mouse. Metabolism. 1992; 41:135-136.

11. Peng, J., Xiao, X., Hu, M., \& Zhang, X. (2018). Interaction between gut microbiome and cardiovascular disease. Life Sciences.

12. Wu ZX, Li SF, Chen H, Song JX, Gao YF, Zhang F, et al. The changes of gut microbiotaafter acute myocardial infarction in rats. PloS one. 2017.

13. Haghikia A, Li XS, Liman TG, Bledau N, Schmidt D, Zimmermann F, et al. Gut Microbiota-Dependent Trimethylamine N-Oxide Predicts Risk of Cardiovascular Events in Patients With Stroke and Is Related to Proinflammatory Monocytes. Arteriosclerosis, thrombosis, and vascular biology. 2018.

14. Suzuki T, Heaney LM, Jones DJ, Ng LL. Trimethylamine N-oxide and Risk after Acute Myocardial Infarction. Clinical chemistry. 2017 63:420-8.

15. Karbach SH, Schonfelder T, Brandao I, Wilms E, Hormann N, Jackel S, et al. Gut Microbiota Promote Angiotensin II-Induced Arterial Hypertension and Vascular Dysfunction. J Am Heart Assoc. 2016.

16. Yan Q, Gu Y, Li X, Yang W, Jia L, Chen C, et al. Alterations of the Gut Microbiome in Hypertension. Frontiers in cellular and infection microbiology. 2017. 
17. Ufnal M, Jazwiec R, Dadlez M, Drapala A,Sikora M, Skrzypecki J. TrimethylamineN-oxide: a carnitine-derived metabolite that prolongs the hypertensive effect of angiotensin II in rats. The Canadian journal of cardiology. 2014.

18. Nagatomo Y, Tang WH. Intersections Between Microbiome and Heart Failure: Revisiting the Gut Hypothesis. Journal of cardiac failure. 2015.

19. Tang WH, Wang Z, Fan Y, Levison B, Hazen JE, Donahue LM, et al. Prognostic value of elevated levels of intestinal microbe-generated metabolite trimethylamine- $\mathrm{N}$-oxide in patients with heart failure: refining the gut hypothesis. Journal of the American College of Cardiology. 2014.

20. Chen K, Zheng X, Feng M, Li D, Zhang H. Gut Microbiota-Dependent Metabolite Trimethylamine N-Oxide Contributes to Cardiac Dysfunction in Western Diet-Induced Obese Mice. Frontiers in physiology. 2017.

21. Zhang H, Meng J, Yu H. Trimethylamine N-oxide Supplementation Abolishes the Cardioprotective Effects of Voluntary Exercise in Mice Fed a Western Diet. Frontiers in physiology. 2017.

22. Collins SM, Bercik P. The relationship between intestinal microbiota and the central nervous system in normal gastrointestinal function and disease. Gastroenterology. 2009; 136(6).

23. Evrensel A, Ceylan ME. The gut-brain axis: The missing link in depression. Clin. Psychopharmacol. Neurosci. 2015; 13(3): 239-244.

24. O'Mahony SM, Clarke G, Borre YE, Dinan TG, Cryan JF. Serotonin, tryptophan metabolism and the brain-gut axis. Behav. Brain Res. 2015; 277: 32-48.

25. Wren AM, Bloom SR. Gut hormones and appetite control. Gastroenterology. 2007; 132(6):2116-2130.

26. Borre YE, O'Keeffe GW, Clarke G, Stanton C, Dinan TG, Cryan JF. Microbiota and neurodevelopmental windows: Implications for brain disorders. Trends Mol. Med. 2014; 20(9): 509-518.

27. Barrett E, Ross RP, O'Toole PW, Fitzgerald GF, Stanton C. gamma-Aminobutyric acid production by culturable bacteria from the human intestine. J. Appl. Microbiol. 2012; 113(2): 411-417.

28. Kimura I, Ozawa K, Inoune D, Imamura T, Kimura K, Maeda T i wsp. The gut microbiota suppresses insulin-mediated fat accumulation via the short-chain fatty acid receptor GPR43. Nat. Commun. 2013; 4: 1829.

29. Perez-Burgos A, Wang B, Mao YK, Mistry B, McVey Neufeld KA, Bienenstock J i wsp. Psychoactive bacteria Lactobacillus rhamnosus (JB-1) elicits rapid frequency facilitation in vagal afferents. Am. J. Physiol. Gastrointest. Liver Physiol. 2013; 304(2): G211-G220.

30. Petra AI, Panagiotidou S, Hatziagelaki E, Stewart JM, Conti P, Theoharides TC. Gutmicrobiota-brain axis and effect on neuropsychiatric disorders with suspected immune dysregulation. Clin. Ther. 2015; 37(5): 984-995.

31. Chrobak AA, Nowakowski J, Dudek D. Interactions between the gut microbiome and the central nervous system and their role in schizophrenia, bipolar disorder and depression. Arch.

32. Dinan TG, Cryan JF. Regulation of the stress response by the gut microbiota: Implications for psychoneuroendocrinology. Psychoneuroendocrinology. 2012; 37(9): 1369-1378. 
33. Dinan TG, Cryan JF. Melancholic microbes: A link between gut microbiota and depression? Neurogastroenterol. Motil. 2013; 25(9): 713-719.

34. Musso G, Gambino R, Cassader M. Obesity, diabetes and gut microbiota: The hygiene hypothesis expanded? Diabetes Care. 2010; 33(10): 2277-2284.

35. Thomas RH, Meeking MM, Mepham JR, Tichenoff L, Posmayer F, Liu S i wsp. The enteric bacterial metabolite propionic acid alters brain and plasma phospholipid molecular species: Further development of a rodent model of autism spectrum disorders. J. Neuroinflammation. 2012; 9: 153.

36. Louis P. Does the human gut microbiota contribute to the etiology of autism spectrum disorders? Dig. Dis. Sci. 2012; 57(8): 1987-1989.

37. Critchfield JW, Hemert van S, Ash M, Mulder L, Ashwood P. The potential role of probiotics in the management of childhood autism spectrum disorders. Gastroenterol. Res. Pract. 2011;161358.

38. Dash S, Clarke G, Berk M, Jacka FN. The gut microbiome and diet in psychiatry: Focus on depression. Curr. Opin. Psych. 2015; 28(1): 1-6.

39. Fremont M, Coomans D, Massart S, De Meirleir K. High-throughput 16S rRNA gene sequencing reveals alterations of intestinal microbiota in myalgic encephalomyelitis/chronic fatigue syndrome patients. Anaerobe. 2013; 22: 50-56.

40. Fond G, Loundou A, Hamdani N, Boukouaci W, Dargel A, Oliveira J i wsp. Anxiety and depression comorbidities in irritable bowel syndrome (IBS): A systematic review and metaanalysis. Eur. Arch. Psychiatry Clin. Neurosci. 2014; 264(8): 651-660.

41. Rao AV, Bested AC, Beaulne TM, Katzman MA, Iorio C, Berardi JM i wsp. A randomized, double-blind, placebo-controlled pilot study of a probiotic in emotional symptoms of chronic fatigue syndrome. Gut Pathog. 2009; 1(1): 6.

42. L. V. Kalia i A. E. Lang, „Parkinson's disease”, Lancet Lond. Engl., t. 386, nr 9996, s. 896-912, sie. 2015.

43. D. W. Dickson i in., „Neuropathology of non-motor features of Parkinson disease”, Parkinsonism Relat. Disord., t. 15, s. S1-S5, grudz. 2009.

44. H. Braak, R. A. I. de Vos, J. Bohl, i K. Del Tredici, ,Gastric $\alpha$-synuclein immunoreactive inclusions in Meissner's and Auerbach's plexuses in cases staged for Parkinson's diseaserelated brain pathology", Neurosci. Lett., t. 396, nr 1, s. 67-72, mar. 2006.

45. K. Kieburtz i K. B. Wunderle, „Parkinson's disease: Evidence for environmental risk factors", Mov. Disord., t. 28, nr 1, s. 8-13, 2013.

46. R. Savica i in., „Medical records documentation of constipation preceding Parkinson disease", Neurology, t. 73, nr 21, s. 1752-1758, lis. 2009.

47. K. M. Shannon, A. Keshavarzian, H. B. Dodiya, S. Jakate, i J. H. Kordower, „Is alphasynuclein in the colon a biomarker for premotor Parkinson's Disease? Evidence from 3 cases", Mov. Disord., t. 27, nr 6, s. 716-719, 2012.

48. F. Scheperjans i in., „Gut microbiota are related to Parkinson's disease and clinical phenotype", Mov. Disord., t. 30, nr 3, s. 350-358, 2015.

49. C. B. Forsyth i in., ,Increased Intestinal Permeability Correlates with Sigmoid Mucosa alpha-Synuclein Staining and Endotoxin Exposure Markers in Early Parkinson's Disease", PLOS ONE, t. 6, nr 12, s. e28032, grudz. 2011. 
50. S. Roy Sarkar i S. Banerjee, „Gut microbiota in neurodegenerative disorders”, J. Neuroimmunol., t. 328, s. 98-104, 152019.

51. I. Rite, A. Machado, J. Cano, i J. L. Venero, „Blood-brain barrier disruption induces in vivo degeneration of nigral dopaminergic neurons", J. Neurochem., t. 101, nr 6, s. 1567$1582,2007$.

52. A. Keshavarzian i in., „Colonic bacterial composition in Parkinson's disease”, Mov. Disord., t. 30, nr 10, s. 1351-1360, 2015.

53. A. Pompei, L. Cordisco, A. Amaretti, S. Zanoni, D. Matteuzzi, i M. Rossi, „Folate Production by Bifidobacteria as a Potential Probiotic Property”, Appl. Environ. Microbiol., t. 73, nr 1, s. 179-185, sty. 2007.

54. S. N. Surwase i J. P. Jadhav, „Bioconversion of 1-tyrosine to 1-DOPA by a novel bacterium Bacillus sp. JPJ", Amino Acids, t. 41, nr 2, s. 495-506, lip. 2011.

55. J. Weller i A. Budson, „Current understanding of Alzheimer's disease diagnosis and treatment", F1000Research, t. 7, lip. 2018.

56. J. M. Hill, C. Clement, A. I. Pogue, S. Bhattacharjee, Y. Zhao, i W. J. Lukiw, „Pathogenic microbes, the microbiome, and Alzheimer's disease (AD)", Front. Aging Neurosci., t. 6, cze. 2014.

57. N. M. Vogt i in., „Gut microbiome alterations in Alzheimer's disease”, Sci. Rep., t. 7, nr 1, Art. nr 1, paź. 2017.

58. M. Elahy i in., „Blood-brain barrier dysfunction developed during normal aging is associated with inflammation and loss of tight junctions but not with leukocyte recruitment", Immun. Ageing, t. 12, nr 1, s. 2, mar. 2015.

59. F. Pistollato, S. Sumalla Cano, I. Elio, M. Masias Vergara, F. Giampieri, i M. Battino, „Role of gut microbiota and nutrients in amyloid formation and pathogenesis of Alzheimer disease", Nutr. Rev., t. 74, nr 10, s. 624-634, paź. 2016.

60. D. Ulluwishewa, R. C. Anderson, W. C. McNabb, P. J. Moughan, J. M. Wells, i N. C. Roy, „Regulation of Tight Junction Permeability by Intestinal Bacteria and Dietary Components", J. Nutr., t. 141, nr 5, s. 769-776, maj 2011.

61. O. Karlsson, E. Roman, A.-L. Berg, i E. B. Brittebo, „Early hippocampal cell death, and late learning and memory deficits in rats exposed to the environmental toxin BMAA ( $\beta$ N-methylamino-l-alanine) during the neonatal period", Behav. Brain Res., t. 219, nr 2, s. 310 320, cze. 2011.

62. A. Asti i L. Gioglio, „Can a Bacterial Endotoxin be a Key Factor in the Kinetics of Amyloid Fibril Formation?”, J. Alzheimers Dis., t. 39, nr 1, s. 169-179, sty. 2014.

63. W. J. Lukiw, „Bacteroides fragilis Lipopolysaccharide and Inflammatory Signaling in Alzheimer's Disease”, Front. Microbiol., t. 7, 2016.

64. E. Akbari i in., ,Effect of Probiotic Supplementation on Cognitive Function and Metabolic Status in Alzheimer's Disease: A Randomized, Double-Blind and Controlled Trial”, Front. Aging Neurosci., t. 8, 2016.

65. A. C. Ouwehand i in., ,Bifidobacterium microbiota and parameters of immune function in elderly subjects", FEMS Immunol. Med. Microbiol., t. 53, nr 1, s. 18-25, cze. 2008.

66. Atkinson MA, Eisenbarth GS, Michels AW. Type 1 diabetes. Lancet 2014;383:69-82. 67. Jamshidi P, Hasanzadeh S, Tahvildari A, et al. Is there any association between gut microbiota and type 1 diabetes? A systematic review. Gut Pathog. 2019;11:49. 
68. Han H, Li Y, Fang J, et al. Gut Microbiota and Type 1 Diabetes. Int J Mol Sci. 2018;19(4):995.

69. Belkaid Y, Hand T. Role of the Microbiota in Immunity and inflammation. Cell. 2014 Mar 27; 157(1): 121-141.

70. Cole D, Bulek A, Dolton G, Schauenberg A, Szomolay B, Rittase W, et al. Hotspot autoimmune $\mathrm{T}$ cell receptor binding underlies pathogen and insulin peptide cross-reactivity. $\mathrm{J}$ Clin Invest. 2016 Jun 1; 126(6): 2191-2204.

71. Leiva-Gea I, Sanchez-Alcoholado L, Martin-Tejedor B, Castellano-Castillo D, Moreno-Indias I, Urda-Cardona A, et al. Gut microbiota differs in composition and functionality between children with type 1 diabetes and MODY2 and healthy control subjects: a casecontrol study. Diabetes Care. 2018;41(11):2385-95.

72. Patterson E, Ryan PM, Cryan JF, et al. Gut microbiota, obesity and diabetes. Postgrad Med J. 2016;92(1087):286-300.

73. Cardwell CR, Stene LC, Joner G, et al. Caesarean section is associated with an increased risk of childhood-onset type 1 diabetes mellitus: a meta-analysis of observational studies. Diabetologia. 2008;51(5):726-735.

74. Woldeamlak B, Yirdaw K, Biadgo B. Role of Gut Microbiota in Type 2 Diabetes Mellitus and Its Complications: Novel Insights and Potential Intervention Strategies. Korean J Gastroenterol. 2019;74(6):314-320.

75. Definition, diagnosis and classification of diabetes mellitus and its complications : report of a WHO consultation. Part 1, diagnosis and classification of diabetes mellitus. [Internet]. Geneva: World Health Organization; c1999 [cited 2020 Aug 18].

76. Sircana A, Framarin L, Leone N, et al. Altered Gut Microbiota in Type 2 Diabetes: Just a Coincidence?. Curr Diab Rep. 2018;18(10):98.

77. Qin J, Li Y, Cai Z, et al. A metagenome-wide association study of gut microbiota in type 2 diabetes. Nature. 2012;490(7418):55-60.

78. Salgaço M, Oliveira L, Costa G, Bianchi F, Sivieri K. Relationship between gut microbiota, probiotics, and type 2 diabetes mellitus. Appl Microbiol Biotechnol. 2019;103(2324):9229-9238.

79. Delzenne NM, Cani PD. Gut microbiota and the pathogenesis of insulin resistance. Curr Diab Rep. 2011;11(3):154-159. doi:10.1007/s11892-011-0191-1 